\title{
The use of feminine elements in the design of modern men's clothing
}

\author{
Lingyun $\mathrm{Wu}^{1, \mathrm{a}}$ \\ ${ }^{1}$ Jiangxi Institute of Fashion Technology, Jiangxi, Nanchang, 330201 \\ a2532197581@qq,com
}

Keywords: Emotional design; CAD; Style; interface

\begin{abstract}
With the development of the apparel industry and the renewal of the computer technology, computer aided design CAD is gradually widely used in the field of fashion design. In today's high working pressure, under the background of the fast pace of life, it is widely hoped to get warm living space. Therefore, this article first introduces the theory of emotional design and its inevitability, analysis of emotional design in software design, the application of intelligent clothing CAD software is discussed in the end of the emotional design, intelligent garment design has a certain guiding significance for the future.
\end{abstract}

\section{Introduction}

National economic rapid development at the same time, people's living standards improve, people in the way I dress more pursuit individuation and high grade, on the basis of the beautiful and comfortable more pursuit of unique style. Since many costume designer clothing enterprise current flow is relatively frequent, any mobility will greatly influence on the establishment of the clothing brand, build based on local adjustment for this change implementation design of database are particularly important. At the same time, the theory of emotional design success in many fields, this paper will analyze the emotional design in the application of CAD software development, making it have more intelligent function.

\section{Summary of emotional design}

The development of emotional design. As early as 1986 in user-centered system design was proposed based on the design of the user that thinks the man-machine interface design should take the user as the center, in line with the user's mental habits, can be more attractive, will greatly improve productivity. With the popularity of computer, more and more people by typing, over time, many people in arthritis, hand out of the cocoon and a series of occupational disease, therefore is proposed based on ergonomics keyboard Angle adjustment. In the end of last century, the "machine for this" design idea hit from the psychology, more and more designers to consider the user's habits and operation habits. Professor Norman link availability and emotion, thus represents the theory of emotional design formally established.

The so-called emotional design refers to the design method based on human psychological needs, denied too much emphasis on product function oriented design concept. Product emotion design can fully meet the demand of consumer or user experience and the emotion, to make it produce psychological pleasure, achieve high emotional compensation. The vitality of emotional design make the product more, can truly reflect the connotation of design.

The necessity of emotional design. Design philosophy in the new period for the development and application of emotional design provide fertile soil, science and technology as the material basis for the emotional design, is the pursuit of spiritual wealth, emotional need the way. The current China's garment industry is still in under the background of modern production, enrich people get just the physical level. From the Angle of social and cultural development, standardization and programming design cannot meet the people to the ideal pursuit of quality of life. So for the designer, life can only be truly mobilize products that meet the emotional needs passion.

In addition to this, a social psychologist from the United States, the personality theorist and comparative psychologist maslow's demand level theory, from the bottom are physiological needs, 
security needs, belonging and love needs, respect needs and self-actualization needs. In clothing design, belonging and love needs, respect needs and self-actualization needs are consistent with emotional design. The widespread use of CAD software to make it function is no longer distinguish between the key to software quality, software designers, from the Angle of psychology, puts forward higher requirements.

The application of emotional design. In recent years, emotional design method is used successfully in multiple fields, achieved satisfactory results. Such as industrial product design, a spider in the shape of a juicer; In the field of user interface design, has a subtle color simplify the operating system, to achieve the effect of vision, perception of beautification; Furniture design field, a figure wall-mounted bookcase into jigsaw puzzle elements. In addition, emotional design also get great progress in the field of software design, the current lack of excellent design of the software product is hard to stand out, when designing software application of emotional design concept can satisfy all customers' needs, these needs include enforceability, ease of use, safety, self healing, etc. Under the background of emotional design, the software must also meet the potential demand of users more, such as user cultural background, leading to the requirement of software demands, specialization, etc. Good software must attach much importance to the demand analysis, make humanized interface design humanization, function, reach a balance between the technique and emotion, make people comfortably enjoy high-tech.

\section{The fusion of clothing CAD software and the emotion design}

With the development of CAD software and the clothing industry upgrade, clothing CAD is experiencing the development of intelligence. Clothing CAD software to network, standardization, personalization, three-dimensional direction. Below will be from two aspects: operation function and interface design of clothing CAD software of the emotional design is analyzed, and represent the level of behavior level and the level of instinct level, two kinds of different levels of design enables users to emotion, experience and cultural aspects of integration, so as to achieve reflection level level, and then accurately reflect the satisfaction of all users of software.

Building campus network has been finished. The whole school has a superior basic network platform, and network resource is abundant. The school has bigger teaching resource database, which includes web-based teaching platform, multimedia teaching attachments, online video books, network question bank, communication forum, E-mail and chat room. And it also has thorough hardware system to support every teaching software running.

\section{Teaching practice of blended learning teaching mode in Photoshop}

Operation function. Function is the user's first impression of a software, software is the basic revelation of practical value. Emotional design can be integrated with the software operating function is stressed to product in exploring the depth of human nature, which allows users to product additional value appreciation, and emotional pleasure. Clothing CAD software development must give customer satisfaction levels of reflection, based on the emotional design CAD software must possess style design, pattern design, grading, nesting, three-dimensional the fitting five systems, the utilization rate were $22 \%, 78 \%, 95 \%, 78 \%$ and $95 \%$. The development of the clothing CAD software should include three levels, respectively, for the computer aided design, the structure of the intelligent two-dimensional intelligent design and three dimensional design. The clothing CAD software on the market at present already meet the demand of the first level; The second level of demand is the end of last century the world scientific research subject; The third layer requirements under the background of $3 \mathrm{~d}$ display technology has a strong practical value.

Style design system. Direction in fabrics, exquisite processing can enhance grid, so as to better simulate fold, the texture of the more prominent and make clothes. Increase transparency design, to simulate the effect of the fabrics such as lace, help designers intuitively felt the characteristics of different fabric texture. Fabric design needs to have large material database support, and provide the user plane accessories on surface and special toolbox. In order to make the characteristics of the 
knitting fabric is more outstanding, can through the aspects such as parameter input more flexible to change the knitting fabric. In style design direction, should establish the design component library and knowledge base, build the design of large curve shape, style name of the database, and completes the synchronization on a regular basis. Will realize the permutation and combination of the elements in the database, can get the number much style collection, intelligent, high automatic degree, also bring more inspiration for the user.

Pattern design, grading, nesting system. Strengthen the automatic playing board function clothing CAD software, users only need to input the key size, choose style, can immediately get the plate type. Prototype system shall be conducted for customer personal data records, and realizes the automatically modify the template, help operators more accurately grasp the difference between sample size and standard sample size. In grading system and discharging system should achieve "self learning" function.

In the interface is beautiful. Beautiful items are usually more can capture user eyeball, garment CAD software interface is beautiful or not, also directly affect the use of the user. From color, layout, ICONS below three aspects to discuss the clothing CAD interface design.

Clothing CAD interface color design. Many people because of the long-term use of garment $\mathrm{CAD}$, garment design from irritation of eyes and discomfort. In order to alleviate clothing CAD to the stimulation of the human eye, can adjust interface background, display the saturation, etc. Icon design can wink more prominent, and the workspace is try to use low brightness and saturation of color: blue, dark green, etc. Should avoid to use warm color as the workspace.

Clothing CAD interface layout design. Users of software interface layout directly affects the overall impression. Based on emotional design of clothing CAD software should conform to the law of people's visual layout, namely the majority of people point of view is from top to bottom, from left to right. People in the face of the computer screen, the upper left corner of the observation is optimal, so the most commonly used software function modules should be installed in the interface of the upper left corner. Kind of the human eye to horizontal motion degree is better than vertical movement, so should avoid top-down movement patterns. Software should give full play to the role of the blank space layout, so as to improve the overall visual sense of balance, also can use white space to better reflect the important information. People in achieving the overall consciousness processing is usually the first transfer the overall consciousness, in line with the gestalt theory. Usually adjacent to stimulate more is seen as a whole. So when the layout in order to make the screen appears in good order, can put the information together, and then improve the layout administrative levels sense, help users faster.

Clothing CAD interface icon design. Before the icon design should focus on interactivity, click, click and click shall, after the state of the different target function module also try to use a different icon. Design clothing CAD icon should be with the aid of pictographic, thereby reducing the user cost of learning. Icon design should be simple, the overall principle, on the basis of the overall simple pursuit of perfect details.

\section{Conclusion}

In this paper, the development of emotional design, inevitability, and elaborates the application situation, and for the application of emotional design in the clothing CAD software is playing well. Combined with related working experience from the level of behavior level and the level of instinct level fusion of emotional design and clothing CAD software, has certain guiding significance for the clothing CAD software of the intelligent.

\section{Reference}

[1] Maochun Wei; Pengpeng li; Guangxin Yan. Based on the analysis of AutoCAD clothing CAD software development advantages [J] textile science and technology progress, 3(2010): 93-95. 
[2] Dongyu Hou; Fengqin zheng. Jianjiang Huang. Reform of the clothing CAD teaching promote the clothing $\mathrm{CAD}$ application [J] journal of hebei university of science and technology (social science edition), 4 (2011) : 23-24.

[3] Yingfen Zhen. The application of clothing CAD system in textile and garment enterprises [J] wind science and technology, 9 (2011) : 71-72. 